\title{
Autoconcepto e identificación social urbana en la ciudad de Copiapó, Chile
}

\section{Self-concept and urban social identification in the city of Copiapó, Chile}

\author{
Ricardo Jorquera Gutiérrez ${ }^{1}$ \\ Universidad Santo Tomás, Copiapó, Chile
}

(Rec: abril 2012; Acep: junio 2012)

\begin{abstract}
Resumen
El siguiente artículo de carácter empírico presenta los principales resultados de una investigación realizada en la ciudad de Copiapó, Chile, en la cual se indagaron los autoconceptos y los procesos de identificación social que sus habitantes poseen hacia la ciudad. Respecto a los autoconceptos se realizó un análisis factorial que arrojó once factores, los cuales fueron comparados por origen, sexo, edad, nivel educativo, nivel de ingresos familiares y estado civil de los encuestados, encontrándose diferencias que permiten describir cómo se estructuran las percepciones de diversos grupos respecto a los copiapinos. Posteriormente, se realizó una regresión lineal múltiple presentando los factores de los autoconceptos como predictores de la identificación social urbana. En tanto, se hizo el mismo procedimiento para observar la relación predictiva de los autoconceptos y de la identificación social urbana sobre la percepción de calidad de vida en la ciudad.

Palabras clave: Autoconcepto, identificación social urbana y percepción de calidad de vida urbana.
\end{abstract}

\begin{abstract}
The following article presents the main empirical results of research conducted in the city of Copiapo, Chile in which self-concepts were investigated and the processes of social identification that its inhabitants have to the city. Regarding self-concepts factorial analysis was performed to throw eleven factors, which were compared by origin, sex, age, educational level, family income level and marital status of respondents, finding that help describe differences are structured perceptions various groups regarding copiapinos. Subsequently, we performed a multiple linear regression showing factors as predictors of self-concepts of urban social identification. Also, the same procedure was performed to observe the predictive relationship of self-concepts and social identification on perceptions of urban quality of life in the city.

Keywords: self-concept, urban social identification and perception of quality of urban life.
\end{abstract}

\footnotetext{
Correspondencia dirigida a: Ricardo Jorquera Gutiérrez. E-mail: rjorquera@santotomas.cl,
} 


\section{Introducción}

\section{Concepto de identidad social e identidad social urbana}

La identidad es una de las principales fuentes de significado y experiencia para las personas. Castells (1998) la entiende como el "proceso de construcción del sentido atendiendo a un atributo cultural, o un conjunto relacionado de atributos culturales, al que se da prioridad sobre el resto de las fuentes de sentido" (p. 28). Desde esta posición, para un individuo determinado o un actor colectivo puede haber una pluralidad de identidades, la cual es una fuente de tensión y contradicción tanto en la representación de uno mismo como en la acción social.

En el caso de la psicología social, en las últimas décadas han sido muy influyentes los aportes de Henry Tajfel para el entendimiento de la identidad social. Para este autor, la identidad social sería la parte del autoconcepto del individuo que deriva del conocimiento de su pertenencia a un grupo social (o grupos sociales) junto con el significado emocional y valorativo asociado a dicha pertenencia (1984). Desde esta perspectiva, en la activación, mantenimiento y cambio de la identidad social actuarían procesos individuales, grupales y macrosociales. Dentro de los procesos individuales se destaca la motivación de la persona para evaluarse a sí misma de forma positiva y la importancia que el grupo tiene para la persona. La combinación de ambos procesos influye en la motivación de la persona para evaluar al grupo de forma positiva. En tanto, dentro de los procesos grupales se encuentran la comparación con otros grupos, proceso que lleva a la distintividad positiva del propio grupo respecto a otros grupos, el cual es la base de la identidad social. En tanto, respecto a los procesos macrosociales, es importante el contexto en el que se establecen las comparaciones entre grupos, ya que este contexto favorece la distintividad social positiva.

En esta línea de análisis, se destaca la teoría de la autocategorización de Turner, la cual, según Morales (2007), tendría tres importantes aportaciones: 1. El proceso de despersonalización, que emerge al categorizarse la persona a sí misma como miembro de su grupo. Cuando esto ocurre, deja de percibirse como alguien único y diferente al resto y se considera igual que el conjunto de personas de su grupo. 2. Los tres niveles de categorización del Yo (interpersonal, intergrupal e interespecies), cuyo funcionamiento es antagónico, es decir, cuando uno de los niveles está operativo, los otros dos quedan inhibidos. 3. Concepto de prototipo. De acuerdo con esta teoría, se define a las personas del propio grupo o de otros grupos en términos del prototipo.

Coherente con las definiciones de Tajfel y Turner, la identidad social urbana es definida por Valera y Pol (1994) como una subestructura de la identidad social, en la cual el referente de la categorización social es el espacio urbano. En este caso, los entornos urbanos pueden ser entendidos como categorizaciones del self en un determinado nivel de abstracción grupal. El sentido de pertenencia a determinadas categorías sociales incluye también el sentido de pertenencia a determinados entornos urbanos significativos para el grupo.

En el presente trabajo se pretende conocer la relación entre los procesos de identificación social con la ciudad con el autoconcepto que sus propias personas tienen de las personas originarias del lugar. Se entiende autoconcepto como las percepciones que tiene el individuo sobre sí mismo (Harter, 1990; en Amezcua y Pichardo, 2000), es la imagen que los individuos tienen de sí, lo cual se refleja en sus experiencias y en los medios en que estas experiencias se significan. De acuerdo con Valdez y Reyes (1992), el autoconcepto se define como una estructura mental de carácter psicosocial, que implica una organización de aspectos conductuales, afectivos y físicos, reales e ideales, acerca del propio individuo, que funciona como un código subjetivo de acción hacia el medio ambiente interno y externo que rodea al sujeto.

\section{Contexto general: Identidad en el contexto latinoamericano}

En lo referente al debate que se ha realizado durante los últimos años acerca de la "identidad latinoamericana", según Larraín (1994) encontramos dos posiciones teóricas Una, de carácter "esencialista", plantea que existe una esencia latinoamericana en nuestro pasado histórico, la cual se encuentra congelada en el mundo indígena, o bien en una fusión mestiza originada en el Barroco americano del siglo XVII. Autores como Pedro Morandé (1984) mantienen esta línea de pensamiento. Por otro lado, existe una corriente que destaca el carácter problemático de la identidad, situándola como una constante búsqueda en la cual conviven las diferentes herencias culturales que hemos recibido y continuamos absorbiendo. En síntesis, según esta segunda posición, la identidad es un proyecto que se construye día a día sin esencias elementales, sino como una superposición de tradiciones, pensamientos e ideologías provenientes de distintas partes del mundo. 
Según Castells (2005), en la actualidad, las identidades religiosas, nacionales, territoriales, étnicas y de género, aparecen como principios fundamentales de autodefinición. Junto a estas identidades fuertes de carácter comunitario, construidas histórica y culturalmente, han surgido también identidades individuales, autoconstruidas en torno a un proyecto personal de carácter electivo. En el caso de América Latina, durante el siglo $\mathrm{XX}$ el principio identitario dominante fue la identidad nacional, sin embargo, en la medida que el Estado se constituyó en los años noventa como agente de la globalización y se desapegó de sus bases sociales tradicionales, la separación entre Estado y nación llevó a una crisis de la identidad como principio de cohesión social. De esta manera, la identidad nacional tiende a ser suplantada por el individualismo legitimado por el mercado y/o por identidades comunitarias más fuertes que la identidad nacional, como la religiosa, étnica o regionalista. En este contexto, una identidad individualista particularmente importante ha sido la identidad familiar, o individualismo familiar, en el que se funde el sustrato comunitario y el proyecto autónomo de existencia, vaciado de todo otro contenido diferente a la afectividad inmediata hacia uno mismo y los suyos.

En Chile, históricamente la identidad colectiva fundamental ha sido la identidad nacional, construida a partir del Estado, la cual ha servido como principio de cohesión interna y como principio de defensa frente al exterior. A partir de los años ochenta la ideología del mercado reemplazó a la nación, y la relación entre Estado y nación fue sustituida por la relación entre individuo y mercado, bajo la dirección del Estado, en base a una nación que se convirtió en referencia puramente ideológica. Se diluyeron también las identidades regionales y locales. En estas condiciones, la identidad se individualizó y fraccionó según las oportunidades que el mercado y el sistema de redistribución ofrecieron a cada uno. Durante los noventa la nación siguió separada del Estado, y este último actuó legitimándose en la construcción gradual de la democracia, en la continuidad del crecimiento económico y en el mantenimiento del mercado como principio común aceptado por toda la sociedad. A partir de finales de los años noventa se observó un aumento en la inseguridad subjetiva y se evidenció un incremento de una ideología eminentemente individualista cercana a la ética protestante: esfuerzo propio, trabajo y educación como vía de mejora individual y colectiva (Castells, 2005).

\section{Contexto particular: Algunas ideas acerca de la identidad Copiapina}

Consistente con el concepto de identidad social urbana de Valera y Pol (1994), basado en la teoría de Tajfel y Turner, se puede conjeturar algunos lineamientos en base a los cuales se estructuraría una identidad social asociada a la ciudad de Copiapó, los cuales se basarían en referencia a algunos aspectos históricos, políticos, económicos, religiosos y sociales.

Sobre los aspectos históricos, ha sido hipótesis de algunos historiadores, que los orígenes de Chile se encontrarían en el Valle de Copiapó, con hitos como la llegada de los españoles en 1536 y con la posesión de Chile más tarde por Pedro de Valdivia. De hecho, hay pasajes retratados por historiadores como Carlos María Sayago (2006). Esta tradición española se fue mezclando con las culturas existentes previamente, como los diaguitas, changos e inca. Posteriormente, durante el siglo XIX, fue de un alto impacto el descubrimiento y explotación del mineral de plata de Chañarcillo, el cual proporcionó grandes recursos económicos a la zona y al país en general. Asociado a este hito se construye la primera línea ferroviaria en Chile, la que unió Copiapó con Caldera, facilitando el traslado de mineral y personas dentro de la región. La existencia del mineral de plata en la zona se suma a otros recursos minerales presentes, tales como el hierro, el oro y la plata, que va configurando dinámicas sociales propias de este sector. En esta región comienzan a formarse familias acomodadas (dueñas de minas) y la clase obrera, a cargo de los procesos propios de la minería. Las clases adineradas contaron con las posibilidades económicas de ampliar sus horizontes y viajar a otros países, lo que permitió que muchos de los miembros de estas familias luego se convirtiesen en la clase ilustrada no sólo de la región, sino que también sus ideas políticas, económicas y sociales influyen en todo el territorio nacional. Es así como resaltan figuras como las de José Joaquín Vallejos y Pedro León Gallo (Villalobos, 2009).

Por otra parte, también asociado a la industria minera, se deben destacar los procesos migratorios, resaltando la inmigración de personas de distintos lugares de nuestro país que llegan a estas tierras en búsqueda de nuevas y mejores perspectivas laborales. En este sentido, la región de Copiapó se ha ido configurando poblacionalmente a partir de la unión de grupos culturales de la zona y del resto del país, que se asentaron en estas zonas, con trabajadores y sus respectivas familias. Estos últimos han llegado con demandas y necesidades, pero además han 
aportado con creencias, hábitos y valores culturales que se han ido mezclando con los de los lugareños. En esta misma línea, pero mucho más reciente históricamente, debemos considerar los procesos migratorios asociados a la agricultura, que implican además trabajos temporales sin mayores derechos laborales, donde los trabajadores se deslizan de una zona a otra en momentos determinados del año. Esta situación laboral ha incidido en distintos ámbitos, que van desde las dinámicas familiares hasta las regulaciones económicas y sociales de la ciudad, debido a que los migrantes y el aumento de la población demandan nuevos servicios y productos, generando también nuevas problemáticas urbanas. Asimismo, otra fuente de referencia para la identidad social de los habitantes de Copiapó serían las tradiciones religiosas, que se plasman emblemáticamente en la figura de la Virgen de la Candelaria. Al considerar lo dicho hasta aquí, se puede ver que Copiapó, desde sus inicios hasta ahora, ha sido testigo y constructor de diversos hechos económicos, políticos, culturales y sociales que deben ser analizados desde la propia experiencia de los habitantes, para así conocer cómo estos antecedentes van configurando una identidad colectiva, entendida como el resultado de identidades individuales que recursivamente se organizan (Larraín, 2001).

A continuación se presentará la metodología del estudio, para pasar posteriormente a los resultados, discusiones y conclusiones.

\section{Método \\ Diseño de investigación \\ La presente investigación utilizó un diseño tran- seccional correlacional, a través del cual se procuró observar y describir las relaciones existentes entre las principales variables de este estudio: autoconceptos, identidad social y percepción de calidad de vida.}

\section{Muestra}

Se ocupó un muestreo no probabilístico intencional, compuesto por 632 habitantes de la ciudad Copiapó (Región de Atacama, Chile). De ellos, un 54,83\% eran mujeres y $45,17 \%$ hombres. Según edad, un $36,71 \%$ se encontraba entre 18 y 29 años, un $36,23 \%$ entre 30 y 44 años, 24,21\% entre 45 y 64 años, y un 2,85\% eran personas de más de 65 años. Según ingresos familiares, un $17,20 \%$ declaró entradas por un monto inferior a
$\$ 250.000$, un $44,43 \%$ se encontraba en un rango de ingresos entre $\$ 250.000$ y $\$ 500.000$, un $27,39 \%$ entre $\$ 500.000$ y $\$ 1.000 .000$, y un $10,99 \%$ poseía un nivel de ingresos superior a \$1.000.000. Según nivel educacional, el 9,97\% de las personas sólo tenía un nivel de estudios básicos, un $52,85 \%$ un nivel de estudios medios, un $25,16 \%$ un nivel de estudios técnico profesional y un 12,03\% poseía estudios universitarios finalizados. Según origen, 51,32\% de las personas de la muestra era nacido en la ciudad de Copiapó y un $48,68 \%$ era originario de otras ciudades.

\section{Técnicas de recolección de datos}

El levantamiento de los datos se realizó mediante un cuestionario de autorreporte compuesto por una escala de autoconcepto, una escala de identificación social y una pregunta de evaluación general de la percepción de calidad de vida en la ciudad. Asimismo, se incorporó un set de preguntas de identificación general (sexo, edad, nivel educativo, nivel de ingresos, nacido en Copiapó, etc.).

Autoconcepto: A los encuestados se les expuso la afirmación "Yo siento que el copiapino es una persona...”, y se les pidió que plantearan si estaban totalmente en desacuerdo, en desacuerdo, de acuerdo o totalmente de acuerdo, con 37 adjetivo calificativos:

\begin{tabular}{lccc}
\hline Tímida & Ordenada & Amable & Cariñosa \\
Humilde & Exigente & Atenta & Traviesa \\
Sumisa & Agresiva & Leal & Inquieto \\
Desconfiada & Rebelde & Respetuosa & Charlatán \\
Resignada & Porfiada & Limpia & Sincera \\
Solidaria & Desobediente & Buena & Activa \\
Ansiosa (nerviosa) & Romántica & Responsable & Servicial \\
Apática & Sentimental & Obediente & Honesta \\
Floja & Simpática & Inteligente & Aplicada \\
Generosa & & & \\
& & &
\end{tabular}

Estos adjetivos fueron extraídos desde una serie de focus groups realizados previamente a la elaboración del presente estudio y desde el cuestio nario de autoconcepto de Valdez-Medina (1994, en ValdezMedina, 1999).

Identificación social urbana: Esta escala estuvo compuesta por 12 items, basados y adaptados desde un cuestionario desarrollado para evaluar la apreciación del espacio realizado por Vidal, Pol, Guàrdia y Peró 
(2004), el cual, entre otras variables, evaluaba el apego y la identificación social. Esta escala demostró una buena confiabilidad (Alfa=0,904). La contestación de los reactivos se realizó mediante una escala lickert de cuatro puntos. Los ítems son los siguientes:

- Me siento parte de Copiapó

- Me gusta vivir en Copiapó

- Tengo intención de continuar viviendo en Copiapó

- Siento Copiapó como algo propio

- Siento Copiapó como parte de mí

- Me siento apegado a Copiapó

- Copiapó es una ciudad muy diferente a otras

- Hay sitios de Copiapó que me recuerdan cosas que me han pasado

- Estoy orgulloso de vivir en Copiapó

- En Copiapó encuentro todo lo que necesito para vivir

- Siento que pertenezco a Copiapó

- Me identifico con Copiapó

Percepción general de calidad de vida: Se incorporó una pregunta con un sistema de contestación en una escala likert de 4 puntos:

- Usted considera que la calidad de vida de los habitantes de Copiapó es...

\section{Procedimientos}

La encuesta se aplicó a sujetos voluntarios en lugares públicos de la ciudad de Copiapó durante el mes de septiembre de 2010. Para ello se entrenó a estudiantes de pregrado de la carrera de psicología, quienes leían las preguntas a los encuestados y registraban las respuestas.

\section{Técnicas de análisis de datos}

El análisis de los datos se efectuó utilizando técnicas estadísticas descriptivas e inferenciales. Dentro de las primeras se realizaron análisis de frecuencia, medidas de tendencia central, especialmente la media y medidas de dispersión, principalmente la desviación estándar. Estas técnicas permitieron realizar análisis reactivos en el caso de los autoconceptos, ítems de identificación social y condiciones de la ciudad.

Se efectuaron también análisis inferenciales multivariados. Los adjetivos con los autoconceptos se agruparon mediante un análisis factorial, utilizando el método de componentes principales y rotación varimax. Luego se realizaron comparaciones de los factores que emergieron por diversos aspectos: sexo, edad, origen, pretensión de seguir viviendo en la ciudad, estado civil, nivel educacional y nivel de ingresos. Estas comparaciones se hicieron utilizando pruebas $t$ de diferencia de medias para muestras independientes y análisis de la varianza de una vía. Las pruebas post hoc se efectuaron mediante la prueba HSD de Tuckey.

El análisis relacional entre las diversas variables del estudio se efectuó mediante procedimientos de regresión lineal múltiples. En el caso de la relación entre el autoconcepto y la identificación social, se utilizó el método introducir. Este mismo método se usó para establecer la relación entre identificación social y percepción de calidad de vida. En el caso de la relación entre los autoconceptos, la identificación social y la percepción de calidad de vida, se utilizó un método de pasos sucesivos.

Todos los cálculos se efectuaron con el soporte del software estadístico SPSS 17.

\section{Resultados}

\section{Autoconcepto}

Los gráficos que se muestran a continuación evidencian los promedios de los adjetivos que resultaron dentro de las diez menciones más importantes, ordenados de mayor a menor, como recuento general, y comparativamente según género y nacidos y no nacidos en Copiapó.

Entre los diez adjetivos que definen con mayor fuerza a los copiapinos, se mezclan tanto calificaciones positivas como negativas. Dentro de las primeras, destaca una visión del copiapino solidario, generoso, bueno e inteligente. Dentro de los adjetivos de carga

Gráfico 1.

Promedio de conceptos respecto a los habitantes de Copiapó.

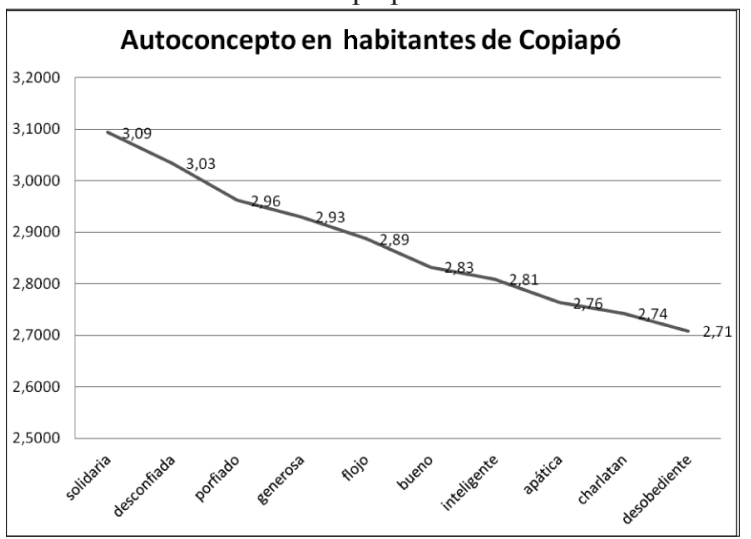


Gráfico 2.

Promedios de conceptos respecto a los habitantes de Copiapó en la muestra de hombres.

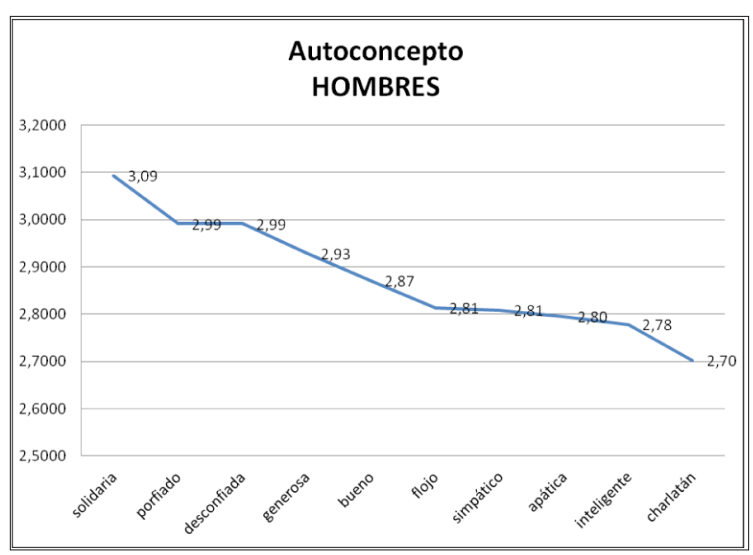

Gráfico 3.

Promedio de conceptos respecto a los habitantes de Copiapó en la muestra de mujeres.

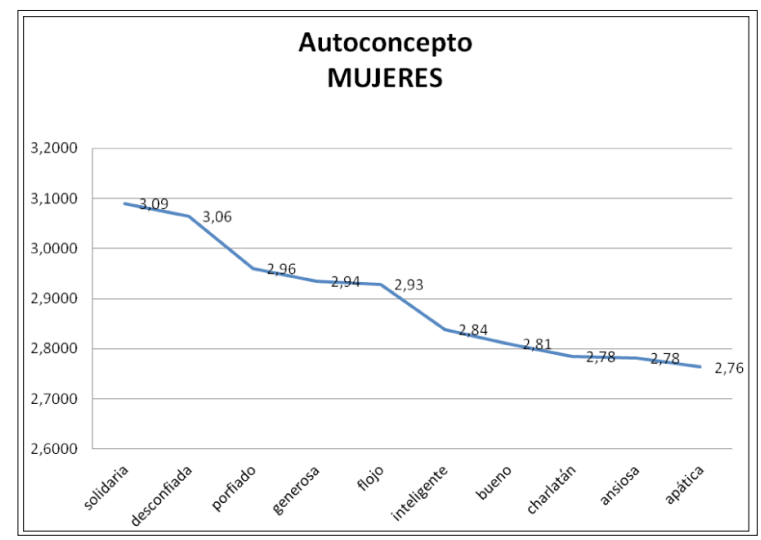

Gráfico 4.

Promedio de conceptos respecto a los habitantes de la ciudad en la muestra de nacidos en Copiapó.

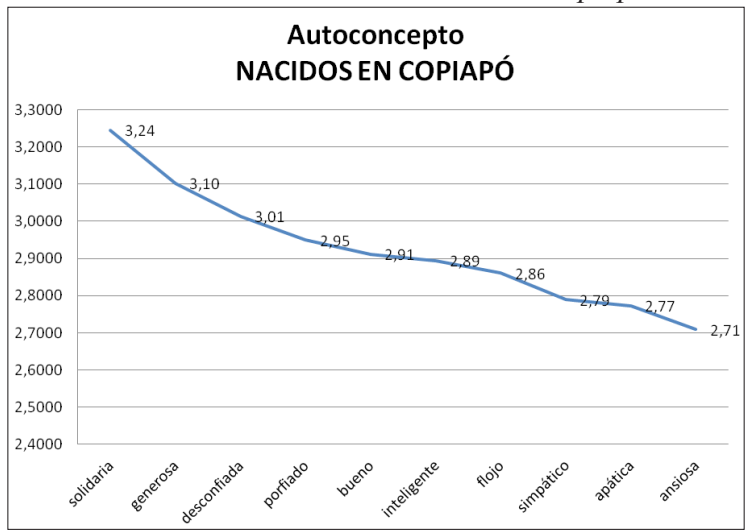

Gráfico 5.

Promedio de conceptos respecto a los habitantes de la ciudad en la muestra de no nacidos en Copiapó.

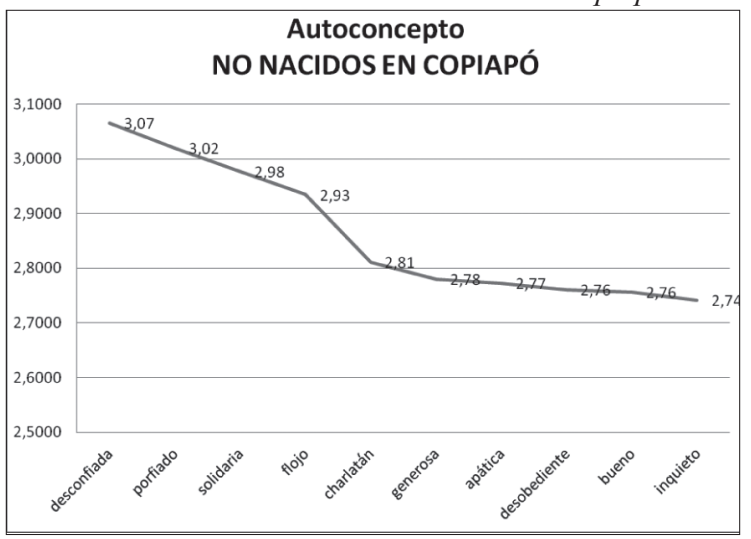

adversa se destacan como desconfiados, porfiados, flojos, apáticos, charlatanes y desobedientes.

Existe una relativa coincidencia entre hombres y mujeres. Esto se evidencia en que de los diez conceptos con mayor fuerza, convergen en ocho: solidarios, porfiados, desconfiados, generosos, flojos, apáticos, inteligentes y charlatanes.

A su vez, entre copiapinos y no copiapinos se converge sólo en cinco de los diez principales conceptos: solidarios, generosos, desconfiados, porfiados y flojos.

Como segundo procedimiento, se efectuó un análisis factorial con el método de componentes principales y utilizando una rotación varimax con los 37 adjetivos. El análisis factorial arrojó 11 factores que explican un $55,65 \%$ de la varianza del instrumento.

Los factores que emergieron de este procedimiento se describen a continuación de acuerdo al nombre con el cual se etiquetó cada factor y los adjetivos que lo constituyeron:

1. Dedicación comprometida: honesto, aplicado, sincero, responsable, activo, leal, servicial.

2. Entrega hacia el otro: generoso, solidario, cariñoso y humilde.

3. Diplomático: inteligente, respetuoso, simpático y bueno.

4. Sensible: romántico, sentimental y ordenado.

5. Suspicaz: desconfiado, porfiado y apático.

6. Activación lúdica: travieso e inquieto.

7. Oposicionismo: rebelde, agresivo y ansiosa.

8. Deferente: atento y amable.

9. Falta de asertividad (pasividad): resignada y charlatán (está último en sentido inverso).

10. Perezoso: flojo.

11. Sometimiento resignado: sumisa y exigente (está último en sentido inverso). 
Los factores anteriores fueron comparados, en primer lugar, según origen (nacidos y no nacidos en Copiapó). Se efectuó una prueba t de diferencia de medias para muestras independientes que arrojó que los nacidos en Copiapó manifiestan una mejor apreciación de los copiapinos en dedicación comprometida $(\mathrm{t}=3,576 ; \mathrm{p}=0.000)$, entrega hacia otros $(\mathrm{t}=5,807$; $\mathrm{p}=0.000)$, sensible $(\mathrm{t}=2,327 ; \mathrm{p}=0.020)$ y suspicaz

Tabla 1.

Matriz de componentes rotados. Análisis factorial de autoconceptos.

Matriz de componentes rotados ${ }^{\mathrm{a}}$

\begin{tabular}{|c|c|c|c|c|c|c|c|c|c|c|c|}
\hline & \multicolumn{11}{|c|}{ Componente } \\
\hline & 1 & 2 & 3 & 4 & 5 & 6 & 7 & 8 & 9 & 10 & 11 \\
\hline honesta & ,635 & & & & & & & & & & \\
\hline aplicado & ,630 & & & & & & & & & & \\
\hline sincero & ,618 & & & & & & & & & & \\
\hline responsable &, 570 & & & & & & & & & & \\
\hline activa &, 550 & & & & & & & & & & \\
\hline leal &, 532 & & & & & & & & & & \\
\hline servicial & ,493 & & & & & & & & & & \\
\hline \multicolumn{12}{|l|}{ obediente } \\
\hline generosa & & ,749 & & & & & & & & & \\
\hline solidaria & & ,709 & & & & & & & & & \\
\hline cariñosa & & ,411 & & & & & & & & & \\
\hline humilde & & ,411 & & & & & & & & & \\
\hline inteligente & & & ,668 & & & & & & & & \\
\hline respetuoso & & &, 526 & & & & & & & & \\
\hline simpático & & & ,485 & & & & & & & & \\
\hline bueno & & & ,449 & & & & & & & & \\
\hline romántico & & & & ,726 & & & & & & & \\
\hline sentimental & & & &, 712 & & & & & & & \\
\hline ordenado & & & & ,485 & & & & & & & \\
\hline desconfiada & & & & & ,672 & & & & & & \\
\hline porfiado & & & & & ,668 & & & & & & \\
\hline apática & & & & & ,494 & & & & & & \\
\hline \multicolumn{12}{|l|}{ desobediente } \\
\hline travieso & & & & & &, 787 & & & & & \\
\hline inquieto & & & & & & ,768 & & & & & \\
\hline rebelde & & & & & & & ,666 & & & & \\
\hline agresivo & & & & & & &, 597 & & & & \\
\hline ansiosa & & & & & & &, 575 & & & & \\
\hline atento & & & & & & & & ,628 & & & \\
\hline amable & & & & & & & & ,608 & & & \\
\hline \multicolumn{12}{|l|}{ limpio } \\
\hline resignada & & & & & & & & & ,679 & & \\
\hline charlatán & & & & & & & & &,- 579 & & \\
\hline \multicolumn{12}{|l|}{ tímida } \\
\hline flojo & & & & & & & & & & ,793 & \\
\hline sumisa & & & & & & & & & & & ,704 \\
\hline exigente & & & & & & & & & & &,- 638 \\
\hline
\end{tabular}

Método de extracción: Análisis de componentes principales.

Método de rotación: Normalización Varimax con Kaiser.

a. La rotación ha convergido en 18 iteraciones. 
$(t=-1,971 ; p=0.049)$. A su vez, quienes pretenden continuar en la ciudad en los próximos cinco años poseen una mejor apreciación de los copiapinos en dedicación comprometida $(\mathrm{t}=2,550 ; \mathrm{p}=0.011)$, pero tienen una apreciación más disminuida en sensible $(\mathrm{t}=-2,086$; $\mathrm{p}=0.037)$ y activación lúdica $(\mathrm{t}=-2,411 ; \mathrm{p}=0.016)$. Por sexo, únicamente se observan dos diferencias significativas: los hombres tienden a manifestar una mejor evaluación de los copiapinos en deferente $(\mathrm{t}=2,299$; $\mathrm{p}=0.022)$ y sometimiento resignado $(\mathrm{t}=3,313 ; \mathrm{p}=0.011)$.

Se realizó una comparación de los factores de autoconcepto según grupos de edad, utilizando el análisis de varianza de un factor, evidenciándose que existirían diferencias significativas en dedicación comprometida $(\mathrm{F}=5,118 ; \mathrm{p}=0,006)$, entrega hacia el otro $(\mathrm{F}=6,992$; $\mathrm{p}=0,001)$, sensible $(\mathrm{F}=3,501 ; \mathrm{p}=0,031)$, oposicionismo $(\mathrm{F}=3,865 ; \mathrm{p}=0,021)$, deferente $(\mathrm{F}=6,637 ; \mathrm{p}=0,001)$, falta de asertividad $(\mathrm{F}=4,321 ; \mathrm{p}=0,014)$ y pereza $(\mathrm{F}=3,719$; $\mathrm{p}=0,025)$. Los contrastes post hoc se efectuaron mediante la prueba de HSD de Tukey. Los resultados más importantes que se extraen de este procedimiento son los siguientes: El grupo más joven (18-29 años), comparado con el grupo de mayor edad (más de 45 años), posee una percepción de los copiapinos como personas con una menor dedicación comprometida, menos oposicionistas y más pasivas y perezosas, aunque más entregadas hacia el otro. A su vez, el grupo de los jóvenes tiende a ver a los copiapinos como personas más sensibles y entregadas hacia el otro en comparación con el grupo de 30 a 44 años. Finalmente, el grupo de mayor edad (sobre 45 años) percibe a los copiapinos como personas más deferentes en comparación al grupo de 30 a 44 años.

Por estado civil se observan diferencias significativas en dedicación comprometida $(\mathrm{F}=3,387 ; \mathrm{p}=0,018)$, entrega hacia el otro $(\mathrm{F}=6,300 ; \mathrm{p}=0,000)$, sensible $(\mathrm{F}=3,451 ; \mathrm{p}=0,016)$, activación lúdica $(\mathrm{F}=4,758$; $\mathrm{p}=0,003)$ y pereza $(\mathrm{F}=7,262 ; \mathrm{p}=0,000)$. Los contrastes post hoc mediante la prueba de Tukey muestran que el grupo de los separados/divorciados evalúan a los copiapinos como personas con una menor dedicación comprometida, comparativamente con la evaluación que realizan los casados y evalúan a los copiapinos menos entregados hacia los otros, en comparación con casados, solteros y viudos; asimismo, los describen como menos sensibles, en comparación al grupo de casados y solteros, aunque menos perezosos, en comparación con casados, solteros y viudos. Por otra parte, los viudos observarían a los copiapinos con mayores niveles de activación lúdica, en relación al grupo de casados, solteros y separados.

Por nivel de ingresos se observan diferencias significativas en entrega hacia el otro $(\mathrm{F}=7,255 ; \mathrm{p}=0,000)$, activación lúdica $(\mathrm{F}=6,924 ; \mathrm{p}=0,000)$, oposicionismo $(\mathrm{F}=2,737 ; \mathrm{p}=0,043)$ y falta de asertividad $(\mathrm{F}=3,103$; $\mathrm{p}=0,026)$. Las pruebas post hoc muestran que el grupo

Tabla 2.

Resultados ANOVA y comparaciones Post Hoc de factores por grupos de edad.

\begin{tabular}{|c|c|c|c|c|c|c|}
\hline \multirow[b]{2}{*}{ Factor } & \multicolumn{3}{|c|}{ Edad } & \multirow[b]{2}{*}{$\mathrm{F}$} & \multirow[b]{2}{*}{$\mathrm{p}$} & \multirow[b]{2}{*}{ Tukey } \\
\hline & 18-29 años (a) & 30-44 años (b) & $45-65$ C & & & \\
\hline 1. Dedicación comprometida & $-0,137$ & 0,002 & 0,184 & 5,118 & 0,006 & $\mathrm{a}<\mathrm{c}$ \\
\hline 2. Entrega hacia el otro & 0,191 & $-0,087$ & $-0,144$ & 6,992 & 0,001 & $a>b, c$ \\
\hline 3. Diplomático & $-0,026$ & 0,078 & $-0,069$ & 1,182 & 0,307 & n.s. \\
\hline 4. Sensible & 0,127 & $-0,117$ & $-0,017$ & 3,501 & 0,031 & $a>b$ \\
\hline 5. Suspicaz & 0,016 & 0,044 & $-0,082$ & 0,825 & 0,439 & n.s. \\
\hline 6. Activación lúdica & $-0,022$ & $-0,055$ & 0,104 & 1,325 & 0,267 & n.s. \\
\hline 7. Oposicionismo & $-0,133$ & 0,032 & 0,139 & 3,865 & 0,021 & $a<c$ \\
\hline 8. Deferente & 0,003 & $-0,158$ & 0,208 & 6,637 & 0,001 & $b<c$ \\
\hline 9. Falta de asertividad (pasividad) & 0,136 & $-0,021$ & $-0,157$ & 4,321 & 0,014 & $a>c$ \\
\hline 10. Perezoso & 0,081 & 0,049 & $-0,177$ & 3,719 & 0,025 & $a>c$ \\
\hline 11. Sometimiento resignado & 0,033 & $-0,033$ & $-0,001$ & 0,252 & 0,777 & n.s. \\
\hline
\end{tabular}


Tabla 3.

Resultados ANOVA y comparaciones Post Hoc de factores por estado civil.

\begin{tabular}{|c|c|c|c|c|c|c|c|}
\hline \multirow[b]{2}{*}{ Factor } & \multicolumn{3}{|c|}{ Estado Civil } & \multirow[b]{2}{*}{ Separ/Divor (d) } & \multirow[b]{2}{*}{$\mathrm{F}$} & \multirow[b]{2}{*}{$\mathrm{p}$} & \multirow[b]{2}{*}{ Tukey } \\
\hline & Soltero (a) & Casado (b) & Viudo C & & & & \\
\hline 1. Dedicación comprometida & $-0,060$ & 0,141 & 0,087 & $-0,299$ & 3,387 & 0,018 & $b>d$ \\
\hline 2. Entrega hacia el otro & 0,075 & $-0,002$ & 0,120 & $-0,587$ & 6,300 & 0,000 & $a, b, c>d$ \\
\hline 3. Diplomático & $-0,066$ & 0,045 & 0,108 & 0,195 & 1,333 & 0,263 & n.s \\
\hline 4. Sensible & 0,053 & 0,008 & 0,052 & $-0,443$ & 3,451 & 0,016 & $a, b>d$ \\
\hline 5. Suspicaz & 0,030 & $-0,029$ & $-0,229$ & 0,051 & 0,643 & 0,588 & n.s \\
\hline 6. Activación lúdica & $-0,008$ & $-0,052$ & 0,723 & $-0,081$ & 4,758 & 0,003 & $\mathrm{c}>\mathrm{a}, \mathrm{b}, \mathrm{d}$ \\
\hline 7. Oposicionismo & $-0,085$ & 0,087 & 0,004 & 0,183 & 1,896 & 0,129 & n.s \\
\hline 8. Deferente & 0,052 & $-0,129$ & 0,020 & 0,238 & 2,469 & 0,061 & n.s \\
\hline 9. Falta de asertividad (pasividad) & $-0,023$ & 0,074 & 0,109 & $-0,252$ & 1,561 & 0,198 & n.s \\
\hline 10. Perezoso & 0,088 & $-0,014$ & 0,120 & $-0,622$ & 7,262 & 0,000 & $a, b, c>d$ \\
\hline 11. Sometimiento resignado & 0,061 & $-0,008$ & $-0,326$ & $-0,217$ & 2,042 & 0,107 & n.s \\
\hline
\end{tabular}

de ingresos inferiores a 250 mil pesos describirían a los copiapinos como personas con una menor entrega hacia el otro y con una mayor activación lúdica, comparativamente con personas de las otras tres categorías de rangos de ingresos, y menos asertivas en comparación con el grupo de ingresos de entre 250 y 500 mil pesos. Junto a esto, se observa que el grupo de entre 250 y 500 mil pesos evalúa a los copiapinos como más oposicionistas que el grupo de entre 500 mil y 1 millón de pesos.

\section{Identificación social urbana}

En relación a la identificación social urbana, se destaca una identificación importante con la ciudad. Un 73,9\% de las personas se siente parte de Copiapó,

Tabla 4.

Resultados ANOVA y comparaciones Post Hoc de factores por grupos de ingresos familiares.

\begin{tabular}{|c|c|c|c|c|c|c|c|}
\hline \multirow[b]{2}{*}{ Factor } & \multicolumn{3}{|c|}{ Ingreso Familiar } & \multirow[b]{2}{*}{$>\mathrm{M} \$ 1000(\mathrm{~d})$} & \multirow[b]{2}{*}{$\mathrm{F}$} & \multirow[b]{2}{*}{$\mathrm{p}$} & \multirow[b]{2}{*}{ Tukey } \\
\hline & $<\mathrm{M} \$ 250$ (a) & $\mathrm{M} \$ 250-\mathrm{M} \$ 500(\mathrm{~b})$ & $\mathrm{M} \$ 500-\mathrm{M} \$ 1000$ C & & & & \\
\hline $\begin{array}{l}\text { 1. Dedicación } \\
\text { comprometida }\end{array}$ & $-0,126$ & $-0,052$ & 0,165 & $-0,028$ & 2,412 & 0,066 & n.s \\
\hline 2. Entrega hacia el otro & $-0,345$ & 0,021 & 0,051 & 0,337 & 7,255 & 0,000 & $\mathrm{a}<\mathrm{b}, \mathrm{c}, \mathrm{d}$ \\
\hline 3. Diplomático & 0,026 & 0,025 & 0,016 & $-0,175$ & 0,789 & 0,500 & n.s \\
\hline 4. Sensible & $-0,191$ & 0,074 & 0,016 & $-0,012$ & 1,859 & 0,135 & n.s \\
\hline 5. Suspicaz & 0,087 & 0,044 & $-0,077$ & $-0,095$ & 1,007 & 0,389 & n.s \\
\hline 6. Activación lúdica & 0,371 & $-0,042$ & $-0,133$ & $-0,160$ & 6,924 & 0,000 & $\mathrm{a}>\mathrm{b}, \mathrm{c}, \mathrm{d}$ \\
\hline 7. Oposicionismo & 0,053 & 0,092 & $-0,177$ & $-0,048$ & 2,737 & 0,043 & $\mathrm{~b}>\mathrm{c}$ \\
\hline 8. Deferente & 0,049 & $-0,013$ & 0,030 & $-0,129$ & 0,528 & 0,663 & n.s \\
\hline $\begin{array}{l}\text { 9. Falta de asertividad } \\
\text { (pasividad) }\end{array}$ & 0,168 & $-0,138$ & 0,066 & 0,034 & 3,103 & 0,026 & $a>b$ \\
\hline 10. Perezoso & $-0,179$ & 0,066 & $-0,073$ & 0,168 & 2,508 & 0,058 & n.s \\
\hline $\begin{array}{l}\text { 11. Sometimiento } \\
\text { resignado }\end{array}$ & $-0,061$ & 0,001 & 0,042 & 0,010 & 0,233 & 0,873 & n.s \\
\hline
\end{tabular}


un $68,5 \%$ dice que siente que pertenece a Copiapó, un $68,2 \%$ dice que le gusta vivir en Copiapó. Se desprende un reconocimiento distintivo de la ciudad, reflejado en preguntas como ¿Copiapó es una ciudad muy diferente a otras?, donde un $85,4 \%$ está de acuerdo o muy de acuerdo, o en "hay sitios de Copiapó que me recuerdan cosas que me han pasado", en donde un $77,9 \%$ está de acuerdo o muy de acuerdo con esta afirmación.

Comparativamente, se observa en esta variable una gran diferencia entre copiapinos y no copiapinos, en donde los primeros tenderían a tener una mayor identificación con la ciudad ( $\mathrm{t}=6,452 ; \mathrm{p}=0,000)$. Por su parte, quienes pretenden continuar en Copiapó los próximos cinco años tienen una mejor identificación con la ciudad que quienes no pretenden hacerlo $(t=9,470 ; p=0,000)$. Por sexo no se observan diferencias significativas $(\mathrm{t}=-1,551 ; \mathrm{p}=0,121)$, por tanto, hombres y mujeres tendrían niveles de identificación equivalentes. Tampoco se observan diferencias significativas por estado civil $(\mathrm{F}=1,745$; $\mathrm{p}=0,157)$, por niveles de ingresos $(\mathrm{F}=1,315 ; 0,268)$, ni por niveles educativos $(\mathrm{F}=0,269 ; \mathrm{p}=0,847)$. Sí se observarían diferencias significativas por edad $(\mathrm{F}=6,364 ; \mathrm{p}=0,002)$. Las pruebas post hoc muestran que los sectores de mayor edad (más de 45 años) tienen mayores niveles de identificación con la ciudad comparativamente con el grupo de personas de entre 18 y 29 años $(\mathrm{p}=0,07)$ y con el grupo de entre 30 y 45 años $(\mathrm{p}=0,03)$.

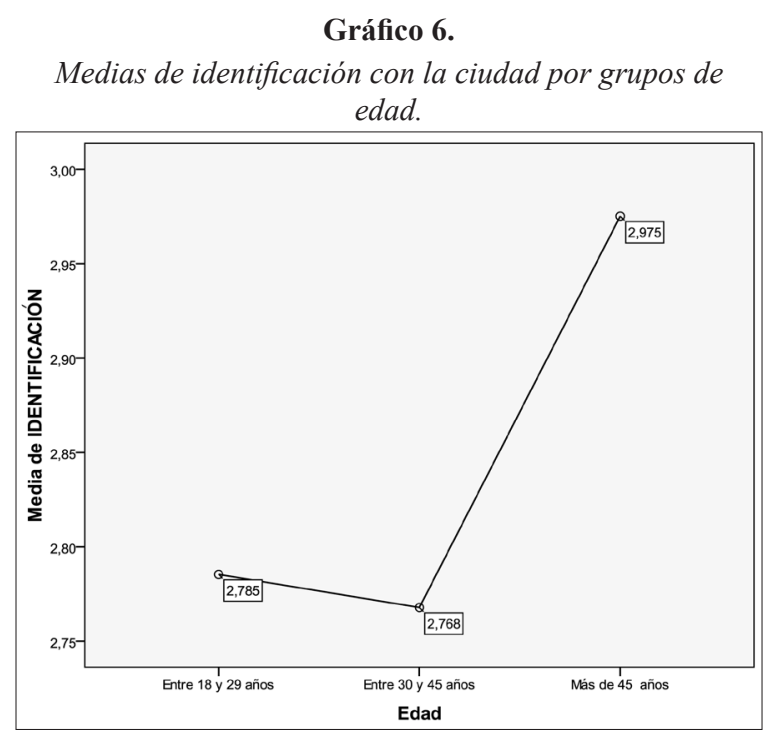

\section{Relación entre autoconcepto e identificación social}

Se estableció un modelo predictivo mediante una ecuación de regresión, utilizando el método introducir. En este caso se ocuparon los once factores de autoconceptos como variables predictoras de la variable criterio identificación social con la ciudad. El modelo resultó ser significativo $(\mathrm{F}=9,559 ; \mathrm{p}=0,000)$ y las predictoras logran explicar un $14,5 \%(\mathrm{R}=0,381)$ de la varianza de identificación social urbana. Las variables que resultaron ser significativas en el modelo son: dedicación comprometida, entrega hacia el otro, diplomático, sensible, oposicionismo y deferente. En el siguiente cuadro y gráfico se logran observar las variables considerablemente relacionadas con identificación social urbana, con el peso de la relación y su significación.

\section{Relación entre autoconcepto, identificación y percepción de calidad de vida urbana}

Se realizó una regresión lineal múltiple mediante el método introducir para observar el comportamiento de los autoconceptos y la identificación social urbana sobre la percepción general de calidad de vida en la ciudad. El modelo resultó ser significativo y las variables predictoras lograrían explicar un $11,7 \%$ de la varianza de percepción de calidad de vida urbana $(F=6,817 ; p=0,000)$. De ellas, las variables que mejor explicarían la percepción de calidad de vida son los autoconceptos: entrega hacia el otro, diplomático, sensible, deferente, sometimiento resignado, oposicionismo y perezoso (estas dos últimas en un sentido inverso). En este modelo no resultó importante la variable identificación social urbana para predecir la percepción de calidad de vida en la ciudad de Copiapó. En el gráfico siguiente se puede apreciar los valores beta y los niveles de significación de cada una de estas variables.

\section{Discusión}

De manera general, se observa que la percepción que tienen los habitantes de Copiapó respecto a los copiapinos es variada, mostrándose una categorización en torno a conceptos tanto positivos como negativos. En este sentido, parece que son conceptos definitorios de ser copiapino, tanto para hombres como para mujeres y para copiapinos y no copiapinos, los calificativos solidarios, generosos, desconfiados, porfiados y flojos. 
Tabla 5.

Coeficientes Beta modelo predictivo autoconceptos (variables predictoras) e identificación social con la ciudad (variable criterio).

Coeficientes $^{\mathrm{a}}$

\begin{tabular}{|c|c|c|c|c|c|c|}
\hline \multirow{2}{*}{\multicolumn{2}{|c|}{$\begin{array}{l}\text { Modelo } \\
\text { B }\end{array}$}} & \multicolumn{2}{|c|}{ Coeficientes no estandarizados } & \multirow{2}{*}{$\begin{array}{c}\text { Coeficientes } \\
\text { tipificados }\end{array}$} & \multirow[b]{2}{*}{$\mathrm{t}$} & \multirow[b]{2}{*}{ Sig. } \\
\hline & & Error típ. & Beta & & & \\
\hline \multirow[t]{12}{*}{1} & (Constante) & 2,829 & 023 & & 121,252 & 000 \\
\hline & Dedicación comprometida &, 076 & 023 &, 121 & 3,244 & 001 \\
\hline & Entrega hacia el otro & 143 & 023 & 228 & 6,141 &, 000 \\
\hline & Diplomático &, 138 & 023 & 220 & 5,929 &, 000 \\
\hline & Sensible &, 054 & 023 & ,086 & 2,323 & 020 \\
\hline & Suspicaz &,- 005 & 023 &,- 008 &,- 213 &, 831 \\
\hline & Activación lúdica &,- 043 & 023 &,- 068 & $-1,835$ &, 067 \\
\hline & Oposicionismo &, 059 & 023 &, 094 & 2,540 &, 011 \\
\hline & Deferente &, 055 & 023 & 087 & 2,340 &, 020 \\
\hline & Falta de asertividad (pasividad) &,- 020 & 023 &,- 032 &,- 872 & ,383 \\
\hline & Perezoso &,- 007 & 023 &,- 010 &,- 281 & ,778 \\
\hline & Sometimiento resignado &, 010 & 023 &, 016 &, 440 & 660 \\
\hline
\end{tabular}

a. Variable dependiente: IDENTIFICACIÓN.

Tabla 6.

Coeficientes Beta modelo predictivo autoconceptos, identificación social con la ciudad (variables predictoras) y percepción general de calidad de vida en la ciudad (variable criterio).

Coeficientes $^{\mathrm{a}}$

\begin{tabular}{|c|c|c|c|c|c|c|}
\hline \multirow{2}{*}{\multicolumn{2}{|c|}{$\begin{array}{l}\text { Modelo } \\
\text { B }\end{array}$}} & \multicolumn{2}{|c|}{$\begin{array}{l}\text { Coeficientes no } \\
\text { estandarizados }\end{array}$} & \multirow{2}{*}{$\begin{array}{l}\text { Coeficientes } \\
\text { tipificados }\end{array}$} & \multirow[t]{2}{*}{$\mathrm{t}$} & \multirow[t]{2}{*}{ Sig. } \\
\hline & & \multirow{2}{*}{$\begin{array}{c}\text { Error típ. } \\
2,284\end{array}$} & \multirow{2}{*}{$\begin{array}{r}\text { Beta } \\
, 120\end{array}$} & & & \\
\hline \multirow{13}{*}{1} & (Constante) & & & & 18,965 &, 000 \\
\hline & Dedicación comprometida & ,038 &, 024 &, 059 & 1,555 &, 120 \\
\hline & Entrega hacia el otro &, 055 &, 025 & ,086 & 2,212 & 027 \\
\hline & Diplomático &, 103 &, 025 &, 160 & 4,122 &, 000 \\
\hline & Sensible &, 060 &, 024 & ,094 & 2,475 &, 014 \\
\hline & Suspicaz &, 021 &, 024 &, 032 &, 849 & ,396 \\
\hline & Activación lúdica &, 010 &, 024 &, 015 & ,407 & 684 \\
\hline & Oposicionismo &,- 099 & 024 &,- 154 & $-4,057$ &, 000 \\
\hline & Deferente &, 090 &, 024 &, 140 & 3,688 &, 000 \\
\hline & Falta de asertividad (pasividad) &,- 010 &, 024 &,- 016 &,- 422 & 673 \\
\hline & Perezoso &,- 058 &, 024 &,- 091 & $-2,402$ &, 017 \\
\hline & Sometimiento resignado &, 050 & ,024 &, 077 & 2,043 &, 041 \\
\hline & IDENTIFICACIÓN &, 063 &, 042 &, 062 & 1,511 &, 131 \\
\hline
\end{tabular}

a. Variable dependiente: Usted considera que la calidad de vida de los habitantes de Copiapó es... 
Esto habla que los habitantes de Copiapó poseerían de manera amplia una visión del copiapino como una persona que se entrega hacia otros con generosidad pero con reservas, y que a la vez mostraría rigidez y poca proactividad frente a acciones productivas.

En esto los copiapinos tendrían una mejor percepción que los no copiapinos. Los copiapinos tienden a verse más honestos, aplicados, sinceros, responsables, activos, leales y serviciales; también más generosos, solidarios, cariñosos y humildes; y más románticos, sentimentales y ordenados; pero menos desconfiados, porfiados y apáticos que la perceoción de los no copiapinos. Esto viene a confirmar los supuestos teóricos de Tajfel (1981; en Morales et al, 2007) respecto a la apreciación positiva. Este autor plantea que un proceso importante en la activación, mantenimiento y cambio de la identidad social es la motivación de la persona para evaluarse a sí misma de forma positiva y la importancia que el grupo tiene para la persona. La combinación de ambos procesos influye en la motivación de la persona para evaluar el grupo de forma positiva. A su vez, existen procesos grupales, como la comparación con otros grupos, proceso que lleva a la distintividad positiva del propio grupo respecto a otros lo cual es la base de la identidad social.

La intención de permanecer o emigrar de la ciudad sería otro factor que se relacionaría con el autoconcepto acerca de los copiapinos. Quienes pretenden continuar en la ciudad poseen una apreciación de los copiapinos como honestos, aplicados, sinceros, responsables, activos, leales y serviciales; pero a su vez los menos románticos, sentimentales, ordenados, traviesos e inquietos que quienes pretenden irse de la ciudad. Es posible conjeturar que quienes permanecen en la ciudad destacarían características del copiapino que los definirían como personas correctas y amables, aunque poco demostrativos en los planos afectivos.

La edad también repercutiría en los autoconceptos. $\mathrm{Si}$ bien los jóvenes tienden a ver a los copiapinos como personas más solidarias, también los aprecian como menos dedicados y comprometidos, y más pasivos y flojos que los grupos más adultos. Esta visión aparentemente ambivalente, permite evidenciar un reconocimiento de los jóvenes hacia los copiapinos como buenas personas pero faltos de dinamismo para atender a los requerimientos del mercado laboral. Es posible hipotetizar que los jóvenes, dada su familiarización con las redes de información mundializadas, estarían más atentos a las nuevas necesidades en capital humano que surgen con los cambios en el terreno económico, por ello, se mostrarían críticos frente a los habitantes de Copiapó en relación a este ámbito, aunque valorizándolos en otros espacios, como en este caso es la solidaridad.

Otro factor que repercutiría en el autoconcepto es el estado civil. En este plano, lo más llamativo de los resultados comparativos es que los separados/divorciados, a pesar de que son el grupo que reconoce menos flojos a los copiapinos, tienden a evaluarlos también como menos honestos, aplicados, sinceros, responsables, activos, leales y serviciales; además menos generosos, solidarios, cariñosos y humildes; $\mathrm{y}$ a su vez menos románticos, sentimentales y ordenados. Cabe acá la pregunta de si en esto tendría alguna influencia la forma de trabajo y producción en la ciudad, pues pareciera que no se cuestionaría la disposición hacia el trabajo de los copiapinos pero sí su compromiso y lealtad, su orientación solidaria hacia los demás, y aspectos en el plano afectivo. Una hipótesis relacional sería, por tanto, que las formas de producción influirían en la separación de las personas, condición que afectaría, en un proceso de generalización de rasgos, a observar de manera amplia a los copiapinos de acuerdo a su propia experiencia de rompimiento. Interesante, y un desafío para futuros estudios, sería indagar en las razones que llevarían a los quiebres de parejas en la región, la influencia de los sistemas productivos y los aspectos socio-psicológicos que estarían a la base de los procesos de ruptura.

Por nivel de ingreso, llama especialmente la atención la tendencia de los sectores de menores recursos a evaluar de manera más negativa a los copiapinos, en donde los caracterizarían, entre otras cosas, con menores niveles de solidaridad y asertividad que los grupos de ingresos más altos. En esto se puede hipotetizar un proceso de separación identitaria endo/exogrupos, en los cuales las personas de menores ingresos se excluirían del grupo copiapinos, identificándose con características diferenciadoras. Es interesante avanzar en la profundización de la identificación urbana en este grupo, pues podría tener repercusiones en la inclusión social de estas personas con la ciudad.

Una segunda línea de indagación estuvo referida al concepto de identificación social. Es preciso mencionar que en la teoría de la Identificación social de Henry Tajfel el autoconcepto se conforma al menos en parte por la identificación con los grupos de pertenencia y de referencia. Esto ocurre a través de tres procesos: categorización, comparación e identificación. Este último elemento tiene que ver con los elementos afectivos, evaluativos, derivados de la adscripción a un determinado grupo o categoría social, y que en definitiva es el 
elemento que completa la identidad social (Tajfel, en González et al, 2005).

Los resultados presentados nos muestran que existe una alta identificación con la ciudad, lo cual se desprende del alto porcentaje que dice sentirse parte de Copiapó (73,9\%), lo que se traduciría también en un agrado de vivir en esta ciudad, pues un $68,2 \%$ dice que le gusta vivir en Copiapó. Las comparaciones efectuadas no muestran diferencias significativas entre los grupos distribuidos por sexo, nivel de ingresos, nivel educacional y estado civil. Sí se encontraron diferencias importantes por origen (copiapinos v/s no copiapinos), intención de continuar viviendo en Copiapó y por edad. En este sentido, los copiapinos y quienes pretenden seguir viviendo allí presentan una mejor identificación con Copiapó que los no copiapinos o quienes no continuarían en la ciudad en los próximos cinco años. Asimismo, se observa que en la medida que aumenta la edad, también se incrementaría el nivel de identificación con la ciudad. Por otro lado, si bien los copiapinos, tienen una identificación mayor que los no copiapinos es interesante observar que existan niveles cercanos y superiores al $70 \%$ en las preguntas referidas a la identificación con la ciudad. Esto a pesar que de la muestra total sólo un $51 \%$ de los encuestados era copiapino. Esto quiere decir que muchos no copiapinos generan un vínculo afectivo con la ciudad, lo cual se traduce en un sentimiento de sentirse parte y apegado con la ciudad, y en definitiva gustarles vivir en Copiapó. Lo anterior es consistente con la relación observada entre pretender quedarse en la ciudad y una mayor identificación con ella. Esto hace hipotetizar dos cosas: 1. En la medida que las personas se identifican con la ciudad, también tendería a disminuir su intención de emigrar a otra; 2. En la medida que no se pretende ir de la ciudad en los años venideros, también tendería a aumentar la identificación con ella.

Se ha observado una asociación entre algunos factores de autoconcepto y de identificación social. Dedicación comprometida, entrega hacia el otro, diplomático, sensible, oposicionismo y deferente. Salvo por el caso del factor oposicionismo, en general prima una relación entre conceptos positivos con la identificación social, es decir, en la medida que se posee una percepción positiva respecto a los habitantes de la ciudad, es posible predecir que exista una adecuada identificación con ella.

La asociación entre los autoconceptos y la identificación social con la percepción de la calidad de vida en Copiapó reduce los factores predictores de este constructo a una serie de autoconceptos. Sin embargo, llama poderosamente la atención la falta de presencia predictiva de la identificación social urbana. Esto hace presumir que la evaluación de la calidad de vida se realiza desde un nivel más cognitivo que emocional (lo mismo sucede con las categorías que conforman los autoconceptos), por ello cobra más importancia la evaluación que se realiza de las personas de la ciudad. En resumen, se puede presumir que las personas evaluarían su calidad de vida en la ciudad y a las personas que la habitan de acuerdo a categorías en donde predominan rasgos cognitivos por sobre los emocionales (como la identificación social por ejemplo). Lo anterior deja en evidencia que los procesos de categorización social, asociados a la identidad social, tienen un carácter cognitivo y la identificación social un carácter evaluativo/ afectivo, tal como lo plantea la teoría de la Identidad social (Tajfel, 1984).

\section{Conclusión}

Para concluir se exponen tres comentarios:

1. Se evidencia la identidad social urbana de los habitantes de Copiapó como un fenómeno que se ha complejizado, alcanzando cada vez más dinamismo. En la actualidad no existiría un concepto homogéneo respecto al concepto que los habitantes de Copiapó poseen de los copiapinos, así como también, el sentimiento hacia la ciudad presenta variabilidades que pueden ser identificadas. Por el contrario, los procesos de identidad social urbana y de identificación social cambiarían en virtud de variables como ser originarios de la ciudad, tener la intención de seguir viviendo en ella, la edad, el estado civil y el nivel de ingresos. Es posible atribuir esta complejidad a los cambios culturales que ha experimentado desde las últimas décadas Chile y el mundo en general, los cuales han sido englobados e identificados por autores como Beck $(2001,2002)$ o Giddens $(1994,1995)$ bajo el nombre de modernidad reflexiva o modernidad tardía. Copiapó se encontraría en un momento en el cual logran convivir (en aparente armonía) grupos con diferentes sistemas de creencias y sentimientos respecto al espacio urbano y sus personas. Por ende, se hace necesario seguir avanzando acerca del entendimiento en ciudades como ésta, de la convivencia de sectores "modernos" y "tradicionales". Es decir, de cómo comparten sistemas ideológicos (representaciones sociales) basados en valores y creencias individualistas, con bajo interés por establecer compromisos fuertes en sus vinculaciones sociales, con sistemas conservadores menos dinámicos, preocupados por 
mantener una noción homogénea de lo que significaría ser copiapino y de lo que en esencia es culturalmente Copiapó. Indiscutiblemente existen aspectos históricos y sociales que determinan las ideas respecto a la ciudad de Copiapó y su gente (la minería y la historia minera, su festividades religiosas y populares, los procesos migratorios, entre otros), sin embargo, estos factores están en la actualidad interactuando con variables macrosociales que complejizan la mirada sobre la identidad local. Sin lugar a dudas, uno de los factores importantes en la generación de una visión más compleja respecto a lo que significa Copiapó y ser copiapino, es la masificación de las tecnologías de la información, las cuales favorecen el intercambio entre sistemas valóricos y de creencias globales, con los que se han construido históricamente en el pasado. Fenómenos que han sido retratados entre otros por Manuel Castell (1998).

2. Desde un punto de vista metodológico, el presente estudio muestra que resulta apropiada la utilización de métodos en base a sistemas estadísticos multivariados para describir percepciones sociales, realizar comparaciones entre grupos y observar el efecto de factores y predictores sobre estas percepciones. La posibilidad de realizar agrupaciones estadísticas de conceptos mediante análisis factorial permite establecer sistemas de representaciones y poder compararlas a través de diversos factores comparativos. De esta manera, se pudo comprender como se estructuran diferencialmente las representaciones respecto a los copiapinos y a su calidad de vida, entendiendo que la vida en la ciudad significa compartir entre personas variadas con percepciones distintas, las cuales conllevan disposiciones motivacionales también distintas hacia la ciudad. A pesar de lo anterior, queda el desafío de indagar una serie de hipótesis que se han planteado, y en ello es sugerible la utilización de técnicas cualitativas de investigación, las cuales son más apropiadas para conocer en profundidad el marco de significados más recónditos de las representaciones y comparaciones efectuados. Se cree que la utilización de sistemas cualitativos de levantamiento y análisis de datos permitiría alcanzar una mejor precisión en las interpretaciones de carácter hipotético que se expresan en este documento.

3. Los resultados que se mostraron en este reporte son exclusivos para entender algunos procesos psicosociales en personas y grupos sociales que habitan en la ciudad de Copiapó. No necesariamente son generalizables a otras ciudades. Queda por tanto el desafío de realizar estudios comparativos en otras ciudades, que favorezcan la validación de los modelos propuestos en este estudio, considerando las diferenciaciones geográficas y culturales. Se recomienda estudiar estos resultados en contextos de aplicación de política urbanística. Se espera que éstos puedan ser útiles para la toma de decisiones en la intervención de la ciudad, tomando como base aspectos psicosociales que predominan en quienes habitan en ella, como lo son los procesos perceptivos y evaluativos del espacio, y los de identidad social.

\section{Referencias}

Amezcua y Pichardo (2001). Diferencias de Género en autoconcepto en sujetos adolescentes. Anales de Psicología, 16 (2), 207-214.

Beck, U. (2001). La reinvención de la política: Hacia una teoría de la modernización reflexiva. En Beck, U., Giddens, A. \& Lash, S. (comps.). La Modernización Reflexiva: Política, tradición y estética en el orden social moderno. Madrid: Alianza.

Beck, U. (2002). La Sociedad del Riesgo Global. Madrid: Siglo XXI.

Castells, M. (1998). La Era de la Información: Economía, Sociedad y Cultura. Alianza: Madrid.

Castells, M. (2005). Globalización, desarrollo y democracia: Chile en el contexto mundial. Fondo de Cultura Económica: Santiago.

Giddens, A. (1994). Consecuencias de la Modernidad. Madrid: Alianza.

Giddens, A. (1995). Modernidad e identidad del Yo: el Yo y la sociedad en la época contemporánea. Barcelona: Península.

González, S., Cavieres, H., Díaz, C. y Valdebenito, M. (2005). Revisión del constructo de Identidad en la Psicología Cultural. Revista de Psicología de la Universidad de Chile, 14 (2), 9-25.

Larraín, J. (1994). La Identidad Latinoamericana: Teoría e Historia. Estudios Públicos, 55, 31-64.

Larraín, J. (2001). Identidad Chilena. Ed. LOM: Santiago.

Morales, F., Gaviria, E., Moya, M. y Cuadrado, I. (2007). Psicología Social. Mc Graw Hill: México.

Morandé, P. (1984). Cultura y Modernización en América Latina. Cuadernos del Instituto de Sociología, Universidad Católica de Chile.

Sayago, C. M. (2006). Historia de Copiapó. Norte Grande Ediciones: Copiapó.

Tajfel, H. (1984). Grupos humanos y categorías sociales. Barcelona: Herder.

Valdez, J. L. y Reyes, I. (1992). Las categorías semánticas y el autoconcepto. México: ELPAC/AMEPSO.

Valera, S. y Pol, E. (1994). El Concepto de la Identidad Social Urbana: Una Aproximación entre la psicología social y la psicología ambiental. Anuario de Psicología, 62 (3), 5-24.

Villalobos, S. (2009). Pedro León Gallo, Minería y Política. Fundación Tierra Amarilla.

Vidal, T., Pol, E., Guàrdia, J. y Peró, M. (2004) Un modelo de apropiación del espacio mediante ecuaciones estructurales. Medio Ambiente y Comportamiento Humano, 5 (1 y 2), 27-52. 